\title{
Contribución al conocimiento de los reservorios del Trypanosoma cruzi (Chagas,1909) en la Provincia de Corrientes, Argentina
}

\author{
Contribution to knowledge of reservoirs of Trypanosoma cruzi \\ (Chagas, 1909) in Corrientes Province, Argentina
}

\author{
María Esther Bar, Benedicta Mabel Alvarez, Elena Beatriz Oscherov, \\ Miryam Pieri Damborsky y Miguel Eduardo Jörg
}

\begin{abstract}
Resumen Con el propósito de identificar a reservorios del Trypanosoma cruzi se investigaron 60 mamíferos en los Departamentos Capital y San Luis del Palmar. Se examinaron: primates, roedores, marsupiales, carnívoros y edentados; 40 vivían en cautiverio y 20 fueron capturados mediante trampas en una comunidad rural forestal. Los mamíferos fueron analizados por xenodiagnóstico, empleándose ninfas de $3^{\circ}$ o $4^{\circ}$ estadío de Triatoma infestans ayunadas durante 2 semanas. Las heces de los triatominos fueron observadas al microscopio (400x) a los 30, 60 y 90 días post-alimentación. En 2 Saimiri sciureus y en 1 Cebus apella se constató infección por tripanosomas cruziformes. Se concluye que la parasitemia detectada fue baja. La presencia de Didelphis albiventris, reservorio potencial de/Trypanosoma cruzi , en una zona de transmisión activa del parásito representa un factor de riesgo, por lo que son necesarias futuras investigaciones epidemiológicas para determinar la real diagnosis de esta parasitosis en la provincia de Corrientes, Argentina.
\end{abstract}

Palabras-claves: Mamíferos reservorios. Trypanosoma cruzi. Xenodiagnóstico. Corrientes.

Abstract In order to identify Trypanosoma cruzi reservoirs in transmission areas, 60 mammals in Capital and San Luis del Palmar Departments, Corrientes, Argentina were studied. Primates, rodents, carnivores, marsupials and edentates were investigated, 40 of them living in captivity and 20 caught with traps in a rural area. The mammals were examined by xenodiagnosis and third or fourth instars nymphs of Triatoma infestans starved for 2 weeks were used. The feces were microscopically observed (400x) for Trypanosoma cruzi infection at 30, 60 and 90 days after feeding. Trypanosoma cruzi-like parasites were identified in 2 Saimiri sciureus and 1 Cebus apella analyzed by xenodiagnosis. It was concluded that parasitemia was low. Howewer, the presence in a forest area of Didelphis albiventris, potential reservoir of the parasite, indicates a risk factor and deserves further epidemiological study for a true diagnosis of this endemic infection .

Key-words: Reservoir mammals. Trypanosoma cruzi. Xenodiagnosis. Corrientes.

Cátedra de Artrópodos. Facultad de Ciencias Exactas, Naturales y Agrimensura. Universidad Nacional del Nordeste, Corrientes, Argentina.

Investigación Financiada por el Consejo Nacional de Investigaciones Científicas y Técnicas.

Dirección para correspondencia: Dra María Esther Bar. FACENA/UNNE, Av. 9 de Julio 1449, 3400 Corrientes, Argentina.

Tel.: 00543 783-424606. Fax: 00543 783-423968.

E-Mail: mebar@exa.unne.edu.ar

Recebido para publicação em 13/4/98. 
La enfermedad de Chagas es una parasitosis endémica en América Latina y una zoonosis ampliamente extendida, siendo la cuarta causa de morbilidad y mortalidad en el continente. La importancia de los mamíferos en el ciclo silvestre del Trypanosoma cruzi es destacada por numerosos autores1 310111219 25. En Argentina fueron halladas Dasypus hybridus y Didelphis paraguayensis parasitadas por T. cruzi en Chaco17 y Santa Fe18 respectivamente. En Córdoba se verificó infección natural en Calomys musculinus 4 , Calomys laucha 20 y Akodon dolores 5 . En Jujuy se detectó a Octodontomys gliroides infectada24 y en Santiago del Estero se cita a Conepatus chinga y a Galictis cuja como reservorios del T. cruzi, comprobando asimismo una alta tasa de infección en Didelphis albiventris28. En Buenos Aires se encontró a Didelphis albiventris y Akodon azarae infectados por el T. cruzien una región geográfica libre de triatominos ${ }^{14}$.

En la provincia de Corrientes se comprobó una alta prevalencia de Trypanosoma sp en Saimiri sciureus y valores significativos de parasitemia en Cebus apella y Alouatta caraya26. El estado actual del tema en esta provincia está en sus etapas elementales contándose con datos escasos y referencias aisladas, cuyos resultados inclusive no pueden confrontarse por el empleo de diferentes técnicas y/o métodos de evaluación.

Lo precedentemente expuesto justifica la necesidad de detectar en qué medida los mamíferos selváticos contribuyen a mantener la endemia en la región.

El objetivo de este trabajo fue conocer a los mamíferos reservorios del T. cruzi presentes en áreas de transmisión y su participación en los ciclos antrópicos y asinantrópicos.

\section{MATERIALES Y METODOS}

Area de estudio. El área investigada comprendida entre $27^{\circ}$ y $28^{\circ}$ de latitud $S$ y $58^{\circ}$ y $59^{\circ}$ de longitud W, pertenece a los Departamentos Capital y San Luis del Palmar (Figura 1) y está localizada en el distrito oriental de la provincia fitogeográfica Chaqueña7. La temperatura media anual es de $21,5^{\circ} \mathrm{C}$, con lluvias abundantes y frecuentes y el clima es húmedo a subhúmedo8.

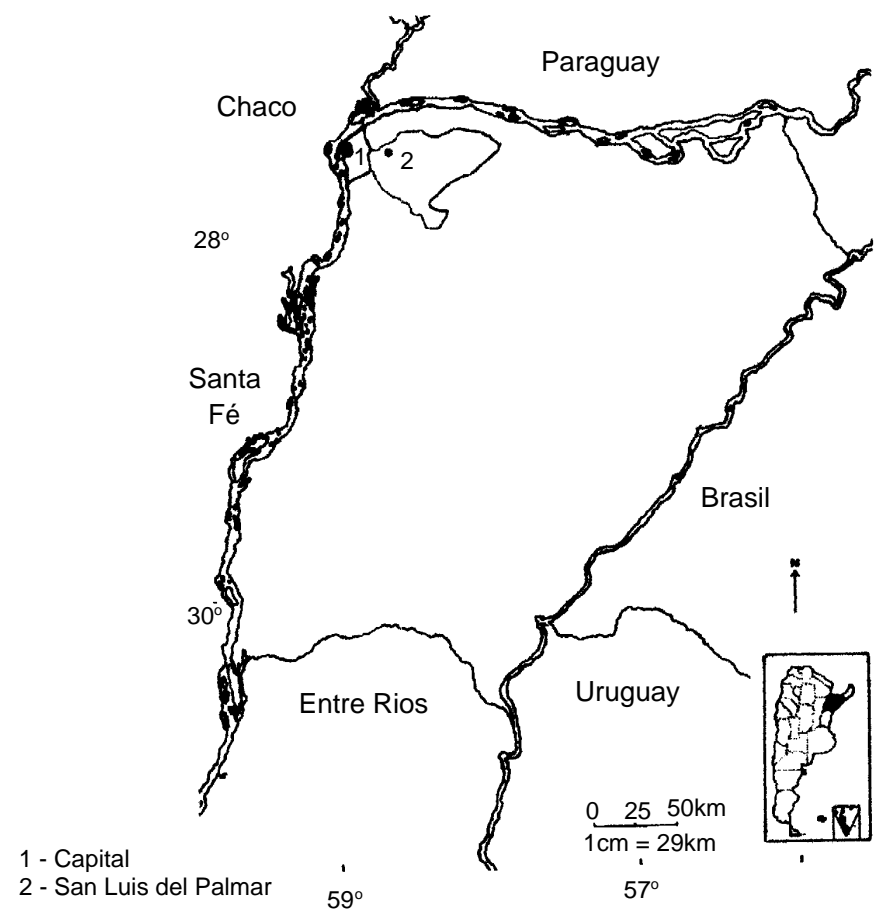

Figura 1 - Localización de los Departamentos Capital y San Luis del Palmar. Provincia de Corrientes. Argentina. 
El paisaje se caracteriza por presentar relieve Ilano con lomadas y esteros. La vegetación dominante es la pradera con especies arbóreas chaqueñas y entrerrianas e higrófilas misioneras en las márgenes fluviales.

Diseño de muestreo. Trampeo. La investigación se realizó en 3 estaciones de muestreo:1) Ralera (Departamento San Luis del Palmar), 2) Dirección de Fauna y Flora de la provincia de Corrientes y 3) el Centro Argentino de Primates (CAPRIM) en San Cayetano, estas dos últimas situadas en el Departamento Capital.

Las estaciones de muestreo fueron seleccionadas en base a la infestación doméstica por Triatoma infestans y a la presencia de seropositivos al $T$. cruzi.

En el área rural forestal de Ralera los mamíferos fueron capturados vivos mediante trampas Tender, National y Sherman utilizándose como cebo: pan frito, fruta, piel de pollo, pescado, harina de maíz con grasa y maní, etc. Fueron seleccionadas 6 unidades y en cada una de ellas se instalaron 3-5 trampas en distintas orientaciones, para aumentar la probabilidad de captura22. Las trampas estaban situadas a distancias que oscilaban entre 100 y $200 \mathrm{~m}$ de las viviendas más próximas y fueron controladas diariamente. El muestreo tuvo una duración de 94 días y fue llevado a cabo en otoño e invierno, dado que estas estaciones representan las de mayor capturabilidad.

Animales en cautividad. Los individuos evaluados en la Dirección de Fauna y Flora provenían de diferentes Departamentos de la provincia de Corrientes. En el Centro Argentino de Primates fueron seleccionados ejemplares nacidos en cautividad y cuyos progenitores de origen boliviano y paraguayo, resultaron negativos al T. cruzien la evaluación serológica practicada para su admisión al Centro.

Xenodiagnosis. Caracterización de reservorios. Cada mamífero fue analizado por xenodiagnóstico, para lo cual fue anestesiado con una inyección intramuscular de acepromacina maleato y clorhidrato de ketamina (Acedán y Ketamina, de Holliday-Scott), cuyas dosis fueron calculadas en función del peso $(2 \mathrm{mg} / \mathrm{kg}$ y $25 \mathrm{mg} / \mathrm{kg}$ de peso respectivamente). Para el xenodiagnóstico se emplearon ninfas de $3^{\circ} \circ 4^{\circ}$ estadio de T. infestans, ayunadas durante 2 semanas, en número de 10 ó 20 según el tamaño de los mamíferos. Las ninfas fueron alimentadas a repleción durante $30-40$ minutos y se conservaron a $28^{\circ} \mathrm{C}\left( \pm 2^{\circ} \mathrm{C}\right)$ y $70 \%( \pm 10 \%)$ de humedad relativa. Las heces de los triatominos diluídas en solución salina $0,85 \%$ fueron observadas $(400 x)$ con un microscopio Carl Zeiss-Standar 25, a los 30, 60 y 90 días post-alimentación 9 .

Para la identificación del T. cruzi fueron utilizados criterios morfológicos, midiéndose largo total, ancho máximo a nivel del núcleo, largo del flagelo libre y cinetoplasto 16 .

Los mamíferos fueron pesados y medidos de acuerdo a procedimientos usuales 29 e identificados sistemáticamente 621 .

La temperatura media del período de muestro fue: $18,0^{\circ} \mathrm{C}$, la mínima: $10,0^{\circ} \mathrm{C}$ y máxima: $29,5^{\circ} \mathrm{C}$. La humedad relativa media fue: $73,2 \%$, la mínima: $56,6 \%$ y la máxima: $87,0 \%$ (Servicio Meteorológico Nacional).

\section{RESULTADOS}

Sesenta mamíferos de diferentes especies fueron investigados por xenodiagnóstico: 40 vivían en cautiverio y 20 fueron atrapados vivos (Tabla 1).

En Ralera se capturaron las especies que se citan a continuación: Akodon azarae, Didelphis albiventris, Bolomys temchuki, Lutreolina crassicaudata, Oxymicterus rufus, Euphractus sexcintus, Chaetophractus vellerosus, Chaetophractus villosus y Myocastor coipo. En ninguno de ellos se verificó infección por T. cruzi.

En Fauna y Flora se analizaron las especies: Dusicyon gimnocercus, Cerdocyon thous, Euphractus sexcintus y Galictis cuja. Tampoco en estos animales se comprobó infección por T. cruzi.
En CAPRIM se evaluaron: Cebus apella, Saimiri sciureus y Alouatta caraya.

Entre los primates del CAPRIM se constató un $8,8 \%(3 / 34)$ de infección por tripanosomas cruziformes, siendo numerosas las formas flagelares observadas en los xenodiagnósticos practicados a 1 ejemplar macho de Cebus apella y 1 macho y 1 hembra de Saimiri sciureus. A los dos años a estos monos se les practicó nuevamente xenodiagnóstico resultando positivos. Las características morfológicas de los tripomastigotes correspondían a las del género Trypanosoma y eran similares a $T$. cruzi.

Del total de mamíferos analizados $73,0 \%$ eran machos y $27,0 \%$ hembras. El peso de los mamíferos pequeños osciló entre: 0,021 y 
$0,050 \mathrm{~kg}$, el de los medianos varió entre 0,700 y $2,700 \mathrm{~kg}$ y los mamíferos de mayor tamaño pesaban 5,0-6,0kg.

En Ralera el $72,2 \%$ de los ejemplares se examinaron en otoño. Los Cricetidae fueron los más capturados ya que representaron el $69,0 \%$ de la población.

Todos los animales de Fauna y Flora se evaluaron durante el otoño y los de CAPRIM durante el invierno.

Tabla 1 - Mamíferos analizados discriminados según orden, familia y especie. Corrientes. Argentina.

\begin{tabular}{|c|c|c|c|c|}
\hline Orden & Familia & Especie & \% de infección & № de animales analizados \\
\hline \multirow[t]{2}{*}{ Marsupialia } & Didelphidae & Didelphis albiventris & 0 & 4 \\
\hline & & Lutreolina crassicaudata & 0 & 1 \\
\hline \multirow[t]{3}{*}{ Edentata } & Dasypodidae & Chaetophractus villosus & 0 & 1 \\
\hline & & Chaetophractus vellerosus & 0 & 1 \\
\hline & & Euphractus sexcintus & 0 & 2 \\
\hline \multirow[t]{3}{*}{ Carnívora } & Canidae & Cerdocyon thous & 0 & 1 \\
\hline & & Ducicyon gimnocercus & 0 & 3 \\
\hline & Mustelidae & Galictis cuja & 0 & 1 \\
\hline \multirow[t]{4}{*}{ Rodentia } & Capromyidae & Myocastor coipo & 0 & 2 \\
\hline & Cricetidae & Akodon azarae & 0 & 6 \\
\hline & & Bolomys temchuki & 0 & 3 \\
\hline & & Oxymycterus rufus & 0 & 1 \\
\hline \multirow[t]{3}{*}{ Primates } & Cebidae & Cebus apella & 3,8 & 26 \\
\hline & & Saimiri sciureus & 28,6 & 7 \\
\hline & & Alouatta caraya & 0 & 1 \\
\hline Total & & & 5 & 60 \\
\hline
\end{tabular}

\section{DISCUSION}

La comunidad de Ralera correspondía a un área de activa transmisión doméstica ${ }^{2}$. Si bien los mamíferos fueron capturados en las proximidades de las viviendas colonizadas por $T$. infestans y con presencia de infectados humanos, los xenodiagnósticos resultaron negativos. Este hecho coincide con lo comprobado en $D$. albiventris atrapados en la cercanía del asentamiento humano de Amamá (Santiago del Estero, Argentina) los que fueron negativos al xenodiagnóstico, a diferencia de los colectados en áreas forestadas, a una distancia de por lo menos $1 \mathrm{~km}$ de la vivienda humana 28 . El resultado obtenido en esta estación de trampeo podría interpretarse como una aparente ausencia de interacción entre el ciclo doméstico y silvestre, dado que no se ha confirmado en $D$. albiventris xenodiagnóstico positivo, lo que indicaría el retorno del parásito al ciclo de transmisión selvática, tal lo verificado en el Estado de Minas Gerais, Brasil13.

No obstante la sóla presencia de $D$. albiventris, reservorio silvestre primario del $T$. cruzi, en la comunidad representa un factor de riesgo para la transmisión, teniendo en cuenta su tendencia a invadir ambientes domésticos y peridomésticos y su susceptibilidad al parásito, como ha sido constatado por otros autores 319.

La ausencia de infección en los mamíferos silvestres restantes podría deberse a un fenómeno similar al antes expuesto para los didelfidos, ya que tanto roedores como dasipodidos fueron positivos al T. cruzi en otras regiones 452023 .

Por otra parte cabría plantear la probabilidad de que los mamíferos de Fauna y Flora habrían sido hallados infectados por T. cruzi en sus hábitats naturales y que después de permanecer en cautiverio la parasitemia se habría negativizado, comportamiento citado por otros investigadores 15 .

Se estima que la especie que parasitaba a los primates era T. cruzi, ya que por una parte tanto $S$. sciureus, como diferentes especies de Cebus fueron encontradas infectadas naturalmente por este tripanosoma 327 y por otra parte la morfología visualizada era coincidente con la descripta en un reporte previo16. 
La positividad del xenodiagnóstico aplicado a los monos a los 2 años plantea la posibilidad de que la parasitemia es persistente en los mismos.

Respecto al origen de la infección no se descarta la transmisión congénita, ya que si bien los progenitores al ingresar al Centro resultaron negativos al T. cruzi, la permanencia en el CAPRIM databa de 10 años al momento de este estudio, período durante el cual podrían haberse infectado. Es probable que la transmisión en cautiverio a sus descendientes se haya dado en forma directa, considerando que la estación de muestreo está localizada en un área con viviendas infestadas con $T$. infestans y donde se constató la presencia de personas seropositivas al T. cruzi.

En la provincia de Corrientes no hay estudios sobre reservorios silvestres del T. cruzi, los datos que existen son aislados y hacen referencia a animales en cautiverio, por lo que la investigación realizada en animales silvestres constituye un aporte original para la provincia de Corrientes, a pesar de la negatividad al $T$. cruzi registrada en ellos. Por lo tanto, se propone como tema de futuras investigaciones conocer su real importancia epidemiológica en la región, así como el aislamiento, caracterización y el estudio electroforético de las isoenzimas del parásito, investigación que no pudo llevarse a cabo por razones operativas.

\section{AGRADECIMIENTOS}

Los autores agradecen a los Dres. J. Ruiz y O.R. Nassiff por habernos permitido trabajar en el CAPRIM y en la Dirección de
Flora y Fauna respectivamente, y a la $\mathrm{Dr}^{\mathrm{a}} \mathrm{C}$. Wisnivesky-Colli por la lectura crítica del manuscrito.

\section{REFERENCIAS BIBLIOGRAFICAS}

1. Alencar JE, Pessoa EP, Sherlock VRA, Tomé GS, Cunha RV. Estudos sôbre a epidemiología da doença de Chagas no Ceará I. Dados preliminares. Revista Brasileira de Malariología e Doenças Tropicais 14:201219, 1962.

2. Bar ME, Oscherov EB, Damborsky MP, Varela ME, Mizdraji G, Porcel E. Triatomismo del Departamento San Luis del Palmar de la provincia de Corrientes, Argentina. Medicina (Buenos Aires) 52:193-201, 1992.

3. Barretto MP. Reservorios del Trypanosoma (Schizotrypanum) cruzi (Chagas 1909). In: Carcavallo RU, Rabinovich JE, Tonn RJ (eds) Factores biológicos y ecológicos en la enfermedad de Chagas. Buenos Aires Argentina: OPS/Servicio Nacional de Chagas, Tomo II, p. 275-288, 1985.

4. Basso B, Eraso AJ, Moretti ER, Albesa I, Kravetz FO. Infección natural de Calomys musculinus (Rodentia, Cricetidae) por Trypanosoma cruzi. Revista de la Asociación Argentina de Microbiología 9:11-16, 1977.

5. Basso B, Moretti E, Albesa I, Eraso A, Kravetz F, D'Alessandro A. Infección natural de Akodon dolores, Thomas, 1916 (Rodentia, Cricetidae) por el Trypanosoma cruzi. Revista do Instituto de Medicina Tropical de São Paulo 24:21-26, 1982.

6. Cabrera A, Yepes J. Mamíferos sudamericanos. Vida, costumbres y descripción. Colección Historia Natural. Ediar. Compañía Argentina de Editores. Buenos Aires, p. 370, 1940.

7. Cabrera AL. Regiones fitogeográficas argentinas. Enciclopedia Argentina de Agricultura y Jardinería II (I). Ediciones Acmé. Buenos Aires, p. 85, 1976.
8. Carnevali R. Fitogeografía de la Provincia de Corrientes. Gobierno de la Provincia de Corrientes. Instituto Nacional de Tecnología Agropecuaria. Corrientes, p. 324, 1994.

9. Cerisola JA, Rohwedder R, Segura EL, Del Prado CE, Alvarez M, De Martini JW. El xenodiagnóstico. Ministerio de Bienestar Social. Buenos Aires. Argentina, p. 127, 1974.

10. Coura JR. Contribução ao estudo da doença de Chagas no estado da Guanabara. Revista Brasileira de Malariología e Doenças Tropicais 18:9-98, 1966.

11. Deane LM. Animal reservoirs of Trypanosoma cruzi in Brasil. Revista Brasileira de Malariología e Doenças Tropicais 16:27-48, 1964.

12. Días JC. Enfermedad de Chagas. Epidemiología Clínica - Terapéutica. Programa de Salud Humana. Buenos Aires, p. 106, 1984.

13. Diotaiuti L, Pereira AS, Loiola CF, Fernandes AJ, Schofield JC, Dujardin JP, Dias JPC, Chiari E. Interrelation of sylvatic and domestic transmission of Trypanosoma cruzi in areas with and without domestic vectorial transmission in Minas Gerais, Brasil. Memórias do Instituto Oswaldo Cruz 90:443-448, 1995.

14. Jörg ME. Didelphis albiventris y Akodon azarae hospedadores del Trypanosoma cruzi, en área geográfica de la provincia de Buenos Aires del vector Triatoma infestans. (Ausencia de lesiones viscerales en los animales). Revista de Ecología Médica y Salud Ambiental. (dados não publicados).

15. Kravetz FO, Eraso A, Moretti E, Albesa Y, Basso B. Variaciones en la prevalencia de tripanosomas semejantes a Trypanosoma cruzi en roedores silvestres. Ecología 3:81-87, 1978. 
16. Mayer HF, Marder G, Galeano RO. Tinción y estudio biométrico de las formas metacíclicas de T. cruzi Chagas, 1909. Revista de Medicina Veterinaria 57:186-190, 1976.

17. Mazza S. Acerca de la infección espontánea de la mulita por el Trypanosoma cruzi en el Norte Argentino. Comprobación en el miocardio y pulmón de los "gigantocitos quísticos" de Magarinos Torres. La Prensa Médica Argentina 17:49-54, 1930.

18. Mazza S, Schreiber F. Hallazgos en el Departamento de Obligado, Santa Fé, de otra especie de mustélido infectado con Schizotrypanum cruzi, de Triatoma infestans infectados en nidos de comadrejas; de Triatoma platensis infectados en nidos de pcitácidos y de Psammolestes coreodes sin infestación en nidos de dendrocoláptidos. Misión de Estudios de Patología Regional Argentina. Universidad de Buenos Aires 34:17-35, 1938.

19. Mello DA. Roedores, marsupiais e triatomíneos silvestres capturados no município de Mambaí-Goiás. Infeção natural pelo Trypanosoma cruzi. Revista Saúde Pública. São Paulo 16:282-291, 1982.

20. Moretti ER, Basso B, Albesa I, Eraso AJ, Kravetz FO. Infección natural de Calomys laucha por Trypanosoma cruzi. Medicina (Buenos Aires) 40 (supl 1):181-186, 1980.

21. Olrog C, Lucero M. Guía de los Mamíferos de Argentina. Ministerio de Cultura y Educación. Fundación Miguel Lillo. Tucumán. Argentina, p. 151, 1981.

22. Peris S. La distribución de los vertebrados terrestres. Curso de doctorado. Universidad de Salamanca 5:61-81, 1987.

23. Romaña C, Schürmann K. La infección espontánea y la experimental del tatú del Chaco santafecino por el Trypanosoma cruzi. Misión de Estudios de Patología
Regional Argentina. Universidad de Buenos Aires 2:969980, 1932.

24. Schweigmann NJ, Alberti A, Pietrokovsky S, Conti O, Riarte A, Montoya S, Wisnivesky-Colli C. A new host of Trypanosoma cruzi from Jujuy, Argentina: Octodontomys gliroides (Gervais and D'orbigny, 1844) (Rodentia, Octodontidae). Memórias do Instituto Oswaldo Cruz. Río de Janeiro 87:217-220, 1992.

25. Telford SR, Tonn RJ. Dinámica de Trypanosoma cruzi en poblaciones de un reservorio primario, Didelphis marsupialis, en los llanos altos de Venezuela. Boletín de la Oficina Sanitaria Panamericana 93:341-364, 1982.

26. Travi BL. Tripanosomiasis en monos americanos. Evaluación de los métodos diagnósticos. Sociedade Brasileira de Primatología. A Primatología no Brasil. In El resumen de los Anales del $2^{\circ}$ Congreso Brasileiro de Primatología, Campinas, p. 489-495, 1985a.

27. Travi BL. Infección natural por Trypanosoma cruzi en Cebus apella. Boletín Primatológico Argentino 3:33-35, 1985b.

28. Wisnivesky-Colli C, Schweigmann NJ, Alberti A, Pietrokovsky SM, Conti O, Montoya S, Riarte A, Rivas C. Sylvatic American trypanosomiasis in Argentina. Trypanosoma cruzi infection in mammals from the Chaco forest in Santiago del Estero. Transactions of the Royal Society of Tropical Medicine and Hygiene 86:38-41, 1992.

29. World Health Organization. Report from the Expert Committee Meeting on Epidemiology of Chagas' disease, Brasilia, July 16-19. Geneva: World Health Organization, mimeographed document TDR/EPICHA/79.1, Rev. 1, p. 25, 1979. 